\title{
A Dynamic Resource Reservation Scheme with Mobility Prediction for Wireless Multimedia Networks
}

\author{
Huei-Wen Ferng, Wen-Yan Kao, David Shiung†, Chien-Liang Liu, Hsing-Yu Chen, and Hung-Yan Gu \\ Department of Computer Science and Information Engineering \\ National Taiwan University of Science and Technology, Taipei 106, Taiwan \\ E-mail: hwferng@mail.ntust.edu.tw \\ $\dagger$ Novatek Microelectronics Corp. \\ 2F, No. 13, Innovation Road I, Science-Based Industrial Park, Hsin-Chu, 300, Taiwan
}

\begin{abstract}
To reduce the call blocking probability (CBP) and call dropping probability (CDP) of real-time and non-real-time traffic in wireless multimedia networks, it is frequent to employ resource reservation to achieve the above-mentioned goal as well as to avoid long latency of path rebuilding. However, pure resource reservation may lead to inefficient resource utilization and increase of CBP and CDP due to frequent handoff in wireless networks. To reduce unnecessary resource reservation, the prediction of moving direction can be incorporated to enhance the pure resource reservation. Therefore, a dynamic resource reservation scheme with mobility prediction is proposed in this paper. In this scheme, a resource request with available range rather than a fixed resource request is specified by each traffic to make the reservation scheme flexible. Using simulation results, we show that mobility prediction can dynamically adjust resource reservation and effectively enhance system resource utilization. Moreover, the available interval of resource request gives flexibility to resource reservation which also improves the system performance.
\end{abstract}

\section{INTRODUCTION}

In the recent few years, high penetration of wireless communication networks have profoundly changed the traditional features of network communications. Accompanying with this technology, resource management is becoming an important issue that draws the attention of network researchers. The most critical problem lies in how to meet the quality of service (QoS) of each connection given some precious network resources. In addition, related problems regarding mobility are also important. When one moves between each neighboring cell, the mechanism of handoff is often an critical factor that influences the QoS.

Another problem accompanying with handoff is that the network resources can not be guaranteed during this process. Aiming at this defect, the protocol RSVP provides an idea to adequately meet the QoS. A novelty of this idea lies in that the server sends a "Path" packet to the client and at the same time gathers the amounts of resources at each hop. The client replies a "Resv" packet immediately after receiving the "Path" packet for each hop. Via this mechanism, one can achieve the end-to-end service guarantee. Since RSVP is originally designed for wireline applications, it is not suitable for wireless communications. Aiming at this feature, some literatures are proposed and may roughly divided by two categories. The first category is to reconstruct the lost and the necessary path, e.g., [2], [3], [5]. The other category is to reserve resources before the beginning of transactions, i.e., [1], [4]. Nevertheless, the CDPs for these techniques during intra-handoff are still too high to be useful. In order to get a better solution, we have to concern the following questions. For what kind of calls should system resources be reserved? How many system resources should be reserved? We thus propose another scheme that can effectively resolve these questions. Our scheme is composed of dynamic resource adjustment, resource reservation, resource reconfiguration, and path prediction. These will be described in the following section.

This remainder of the paper is organized as follows. In Section II, we detail the operations of our proposed scheme. In Section III, we present the simulation results. Finally, conclusions are drawn in Section IV.

\section{The Proposed Scheme}

Let us now present the detailed operations of our scheme through the following components.

\section{A. Basic Resource Reservation}

The strategy of resource reservation is based on the concept of active and passive resource reservation strategy plotted in Fig. 1. A mobile device informs the server two events: the required QoS and the MSpec according to the data type and moving speed of the mobile device, where MSpec contains the information about the region where the mobile device may move to. The larger the region is, the more passive resources will be reserved. This is a trade-off between service reliability and utilization of resources. Evidently, a fixed MSpec is not a clever design; nevertheless, an adaptive MSpec that depending on the moving speed is a better design. When the speed 
is larger, MSpec should accordingly be enlarged. When the mobile device departs from the region defined by MSpec, the originally reserved passive resources should be released to retain better resource utilization.

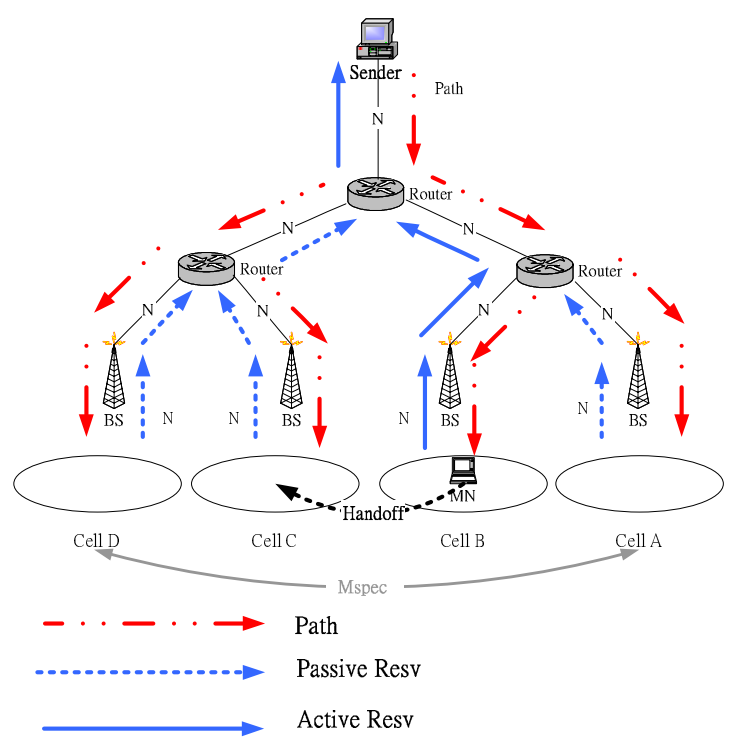

Fig. 1. Basic resource reservation.

\section{B. Dynamic Resource Arrangement}

The strategy of dynamic resource arrangement is adaptively adjusted according to applications, namely, wireline transmission and wireless transmission. In the following, we describe the algorithms of dynamic resource arrangement for the wireless transmission only.

The algorithm may be divided into two categories, namely, requests for new calls and requests for handoff calls. Let us first elaborate on the requests of new calls. When a real-time service is requested, two items $\operatorname{Res}_{1}^{u}$ and $\operatorname{Res}_{1}^{l}$ are defined first. Res $s_{1}^{u}$ resembles the upper bound of used resources; however, $\operatorname{Res}_{1}^{l}$ denotes the according lower bound. When the server cannot supply this upper bound, it will judge if the according lower bound $\operatorname{Res}_{1}^{l}$ can be accepted. If it is acceptable, the system will provide these resources for this real-time application. If it still can not offer the lower bound at this moment, then the server will search if there is any unused reservation. If "yes", the system will judge if it can provide the amount $R e s_{1}^{u}$ or $R e s_{1}^{l}$. If it still can not offer the lower bound, then this request will be rejected. When a nonreal-time application is requested, two items $\operatorname{Res}_{2}^{u}$ and $\operatorname{Res}_{2}^{l}$ will be defined, too. Similar to the real-time application, $\operatorname{Res}_{2}^{u}$ and $R e s_{2}^{l}$ denote the upper bound and the lower bound of the resources, respectively. The following procedures are the same as those of the real-time application.

As for handoff requests, the server will first search for the available resources. If these resources are not enough, the server will reduce the resources used by the non-real-time applications to the lower bound $\operatorname{Res}_{2}^{l}$. Nevertheless, if the resources are still not enough to use, the handoff procedures for both real-time and non-real-time services will be dropped.

\section{Resource Relocation}

After dynamic resource adjustment, the quality of service still can not be guaranteed. It is thus necessary to relocate resources after dynamic resource arrangement. In this subsection, we introduce five viable strategies for resource relocation that may further enhance the system performance.

- Fair sharing (FS): This is the simplest way to relocate resources. Fair sharing is useful when resources are abundant.

- Higher priority for the real-time application or realtime first (RTF): This strategy may achieve better QoS for real-time applications and may be useful for a network that mainly consists of real-time traffic.

- Higher priority for the non-real-time application or non-real-time first (NRTF): Clearly, this strategy is useful in a network that mainly consists of non-real-time traffic. Combining this strategy with dynamic resource adjustment, one can achieve better resource utilization for both real-time and non-real-time applications.

- Fastest leave serves first (FLSF): When this strategy is used, the service departing fastest is served first. The benefit of this idea is that the resources occupied can be released immediately after the service is completed. However, this strategy does not consider types of applications.

- Proportional sharing (PS): This strategy assigns the available resources according to the ratio of the amount of real-time traffic and the amount of non-real-time traffic. After this, perform strategy of higher priority for the fastest departure application mentioned above. This strategy is a compromise of both fairness and resource utilization.

\section{Path Prediction}

Utilizing the strategies of basic resource reservation and dynamic resource adjustment, we may optimize the resource utilization and avoid dropping of service. In order to use the resource in a more efficient way, we add the mechanism of path prediction in our scheme.

Our scheme is to set up passive reservation under the region defined by MSpec and avoid service dropping during handoff. Every passive reservation may not actually be used. Thus, these unused reservations are a burden of the system. To remove the unnecessary burden of the system, we utilize the path prediction to estimate where the mobile device will move to and to determine the size of MSpec in an accurate manner. In the following, we details the mechanism of the path prediction.

- Moving model of users: The moving model employed in this paper is based on that proposed in [6]. The initial position of a mobile device in a cell is randomly distributed. Its moving speed and moving direction are 
TABLE I

PARAMETER SETTING OF USER MOBILITY

\begin{tabular}{|c|c|}
\hline & High speed \\
\hline parameter & value \\
\hline$V_{\max }$ & $70 \mathrm{~km} / \mathrm{hr}$ \\
\hline$\Delta v$ & uniformly distributed on $[-10,10]$ in $\mathrm{km} / \mathrm{hr}$ \\
\hline$\Delta \phi$ & uniformly distributed on $\left[-\frac{\pi}{12}, \frac{\pi}{12}\right]$ \\
\hline$p$ & uniformly distributed on $[0,1]$ \\
\hline \multirow[t]{2}{*}{$p_{s}$} & 0.9 \\
\hline & Medium speed \\
\hline parameter & value \\
\hline$V_{\max }$ & $50 \mathrm{~km} / \mathrm{hr}$ \\
\hline$\Delta v$ & uniformly distributed on $[-5,5]$ in $\mathrm{km} / \mathrm{hr}$ \\
\hline$\Delta \phi$ & uniformly distributed on $\left[-\frac{\pi}{8}, \frac{\pi}{8}\right]$ \\
\hline$p$ & uniformly distributed on $[0,1]$ \\
\hline \multirow[t]{2}{*}{$p_{s}$} & 0.7 \\
\hline & Low speed \\
\hline parameter & value \\
\hline$V_{\max }$ & $30 \mathrm{~km} / \mathrm{hr}$ \\
\hline$\Delta v$ & uniformly distributed on $[-3,3]$ in $\mathrm{km} / \mathrm{hr}$ \\
\hline$\Delta \phi$ & uniformly distributed on $\left[-\frac{\pi}{4}, \frac{\pi}{4}\right]$ \\
\hline$p$ & uniformly distributed on $[0,1]$ \\
\hline$p_{s}$ & 0.5 \\
\hline
\end{tabular}

changed every time period $\Delta t$. The new moving speed $v_{\text {new }}$ is determined by

$v_{\text {new }}= \begin{cases}\min \left\{\max \left[v_{\text {old }}+\Delta v, 0\right], V_{\text {max }}\right\}, & \text { if } p \leq p_{s}, \\ 0, & \text { otherwise, }\end{cases}$

where $v_{\text {old }}$ means the old speed, $\Delta v$ is the step size of speed, $V_{\max }$ is a pre-specified maximum of speed, $p$ represents a probability, and $p_{s}$ is a pre-specified probability threshold. As for the moving direction, it is uniformly distributed. Thus, the moving direction is determined by

$$
\phi_{\text {new }}=\phi_{\text {old }}+\Delta \phi,
$$

where $\phi_{\text {new }}\left(\phi_{\text {old }}\right)$ represents the new (old) direction and $\Delta \phi$ denotes the step size of direction. In Table 1, we gives the moving parameters to be used in our simulations. These parameters are divided into three groups, namely, high speed, medium speed, and low speed.

- Method of the path prediction: The method of the path prediction we use here is mainly determined by the relative distances between the mobile device and its neighboring base stations. When the mobile device continues moving, its relative distances to the neighboring base stations are changed, too. In this paper, we use a square cell as shown in Fig. 2. We set a threshold for distance. If the distance between the mobile device and a specific base station is less than this threshold, we may consider that the mobile device is moving toward this direction. The resources in this particular cell can thus be passively reserved. This idea may effectively enhance the utilization of the system resources and reduce the burden of the system since not all neighboring cells should reserve resources for the mobile device.

In Fig. 3, the resultant procedure of our scheme is sketched.

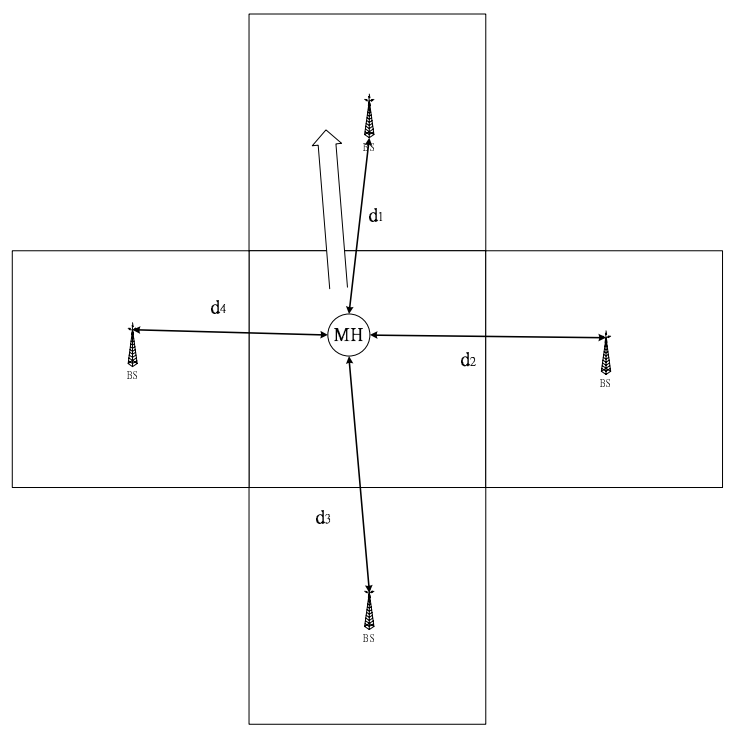

Fig. 2. Cell structure and the prediction of moving direction using the relative distance.

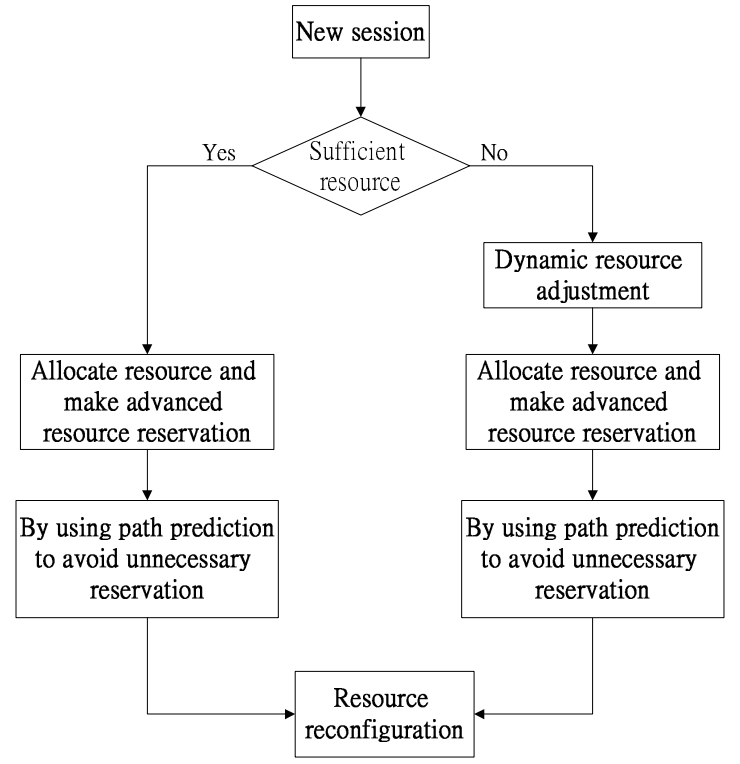

Fig. 3. The procedure of the proposed dynamic resource reservation.

\section{NumericAl Results AND Discussions}

In this section, we investigate impacts of various strategies on service rejection and service dropping through simulations.

\section{A. Models of Simulation}

The data types simulated are divided into two types: realtime and non-real-time applications. We set the data rate for the real-time application higher than the non-real-time one. In this paper, an $8 \times 8$ wrapped around mesh cell structure is assumed. In our simulation, there are a total of 64 mobile agents (MAs) . All of these MAs are connected to the same gateway mobile agent (GMA). The moving direction in the simulation is determined based on (2), while the moving speed is determined based on (1). Handoff is also taken into account 
in our simulation. The other parameters used in the simulation are stated as follows.

1) The input process of the mobile device to the system is a Poisson process with rate $\lambda$.

2) The service time of the mobile device follows an exponential distribution with parameter $\mu$.

3) The system capacity is denoted by $C$.

4) The system load/workload $\rho$ is equal to $\lambda /(C \mu)$.

In the following, we observe system performance in terms of CBP and CDP, where CBP is defined as the probability that a mobile device can not be served and CDP is defined as the probability that a service is dropped by a base station during the handoff process.

\section{B. Simulation Results}

Figs. 4 and 5 are the simulation results of overall (both real-time and non-real-time) CBP and CDP versus workload for various strategies. Obviously, no reservation results in the highest CBP and CDP. These two measures are then improved by the previously proposed HMRSVP. Our proposed dynamic resource reservation (DRR) further gains better performance since flexibility and adjustability are added. Accompanying with the path prediction, the system performance exhibits best as shown in these figures. To have a deeper insight into CBPs of real-time applications and CBPs of non-real-time applications, one can see Figs. 6 and 7 for details.

As for the impacts of various moving speeds on the CBP and CDP performance, they are shown in Figs. 8 and 9, respectively. We note that the size of MSpec varies as the moving speed changes. Specifically, our scheme still maintains lower CBPs/CDPs even in a high speed moving environment.

In Figs. 10 and 11, we study different resource relocation strategies on the system performance. From these figures, we know that RTF provides better QoS guarantee for real-time applications, while NRTF, FLSF, and PS have lower CDP and CBP performance for non-real-time applications as compared to other strategies.

\section{CONCLUSIONS}

In this paper, we propose a dynamic resource reservation mechanism utilizing dynamic resource adjustment with path prediction. In such a scheme, the dynamic resource adjustment adds flexibility to the reservation mechanism. Moreover, the path prediction is further used to predict the proper size of MSpec in order to perform more accurate passive resource reservation, thus avoiding unnecessary bandwidth reservation. These ideas indeed improve the QoS of a mobile device and achieve better resource utilization in the multimedia network. Compared with other competitive counterparts through simulations, our scheme indeed performs better than other existing schemes.

\section{REFERENCES}

[1] M. S. Kim, Y. J. Suh, Y. J. Kim, and Y. Choi, "A resource reservation protocol in wireless mobile networks," in Proc. IEEE International Conference on Parallel Processing Workshops '01, pp. 429-434, Sept. 2001.
[2] B. Moon and H. Aghvami, "Reliable RSVP path reservation for multimedia communications under an IP micromobility scenario," IEEE Wireless Communications, vol. 9, no. 5, pp. 93-99, Oct. 2002.

[3] S. Paskalis, A. Kaloxylos, E. Zervas, and L. Merakos, "An efficient RSVPmobile IP interworking scheme," Mobile Networks and Applications, vol. 8 , no. 3, pp. 197-207, June 2003.

[4] A. K. Talukdar, B. R. Badrinath, and A. Acharya, "MRSVP: a resource reservation protocol for an integrated services network with mobile hosts," Wireless Networks, vol. 7, no. 1, pp. 5-19, January 2001.

[5] C. C. Tseng, G. C. Lee, R. S. Liu, and T. P. Wang, "HMRSVP: a hierarchical mobile RSVP protocol," Wireless Networks, vol. 9, no. 2, pp. 95-102, March 2003.

[6] J. Ye, J. Hou, and S. Papavassiliou, "Integration of advanced reservation and bandwidth reconfiguration based admission control in wireless networks with multimedia services," in Proc. IEEE ICDCS '03, pp. 844-849, May 2003.

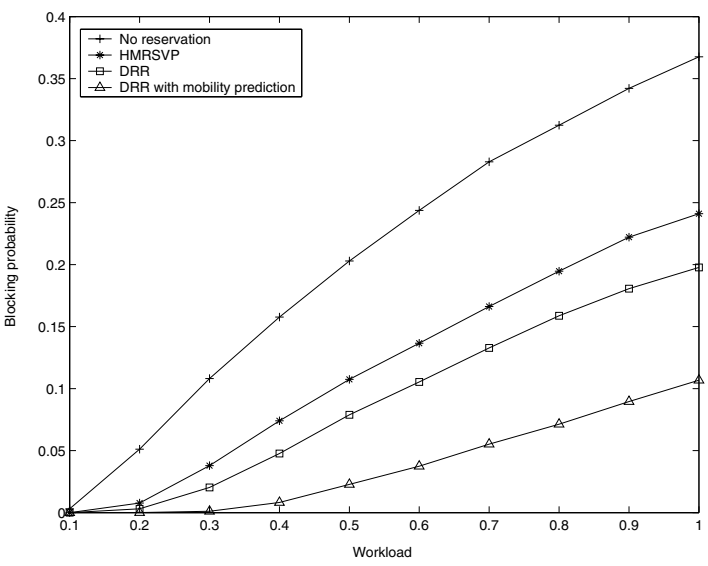

Fig. 4. CBP vs. workload for various strategies.

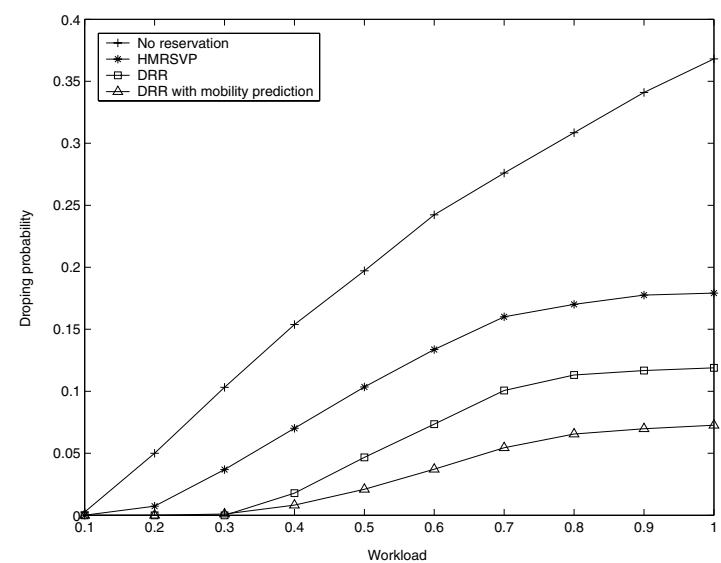

Fig. 5. CDP vs. workload for various strategies. 


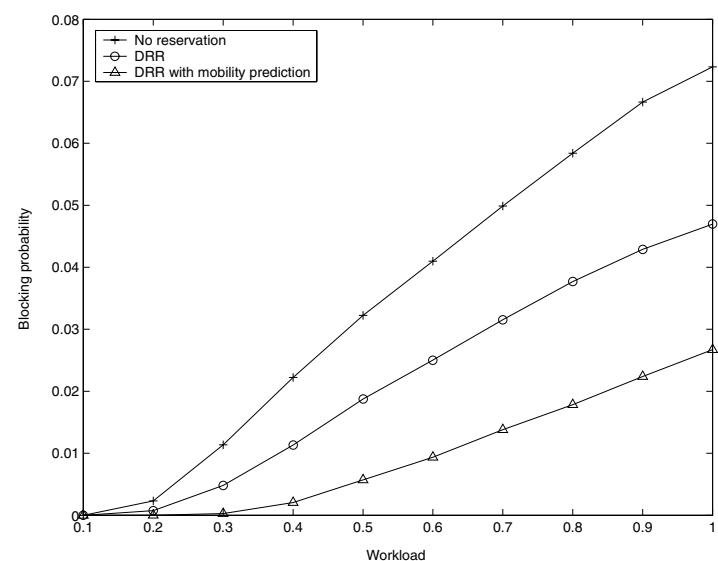

Fig. 6. CBP for the real-time application.

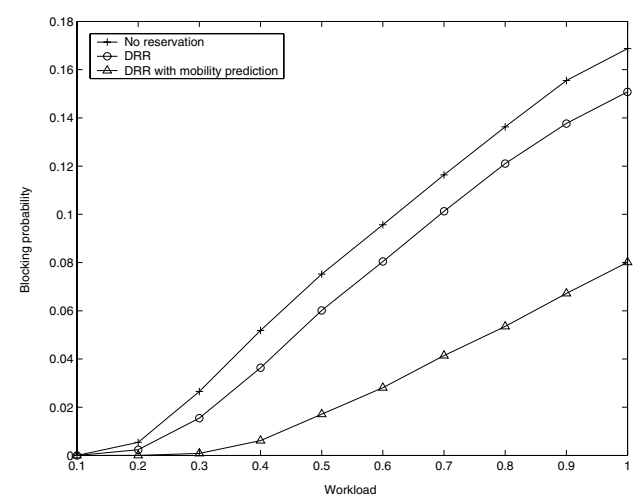

Fig. 7. CBP for the non-real-time application.

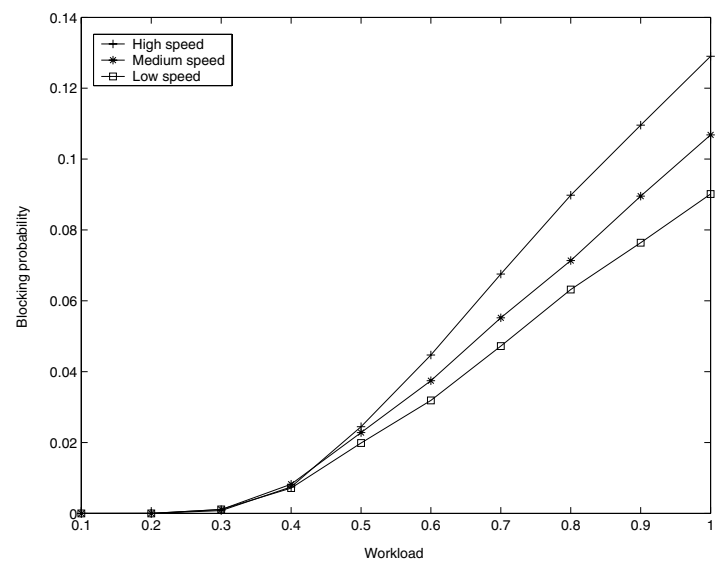

Fig. 8. Impacts on CBP by different moving speeds.

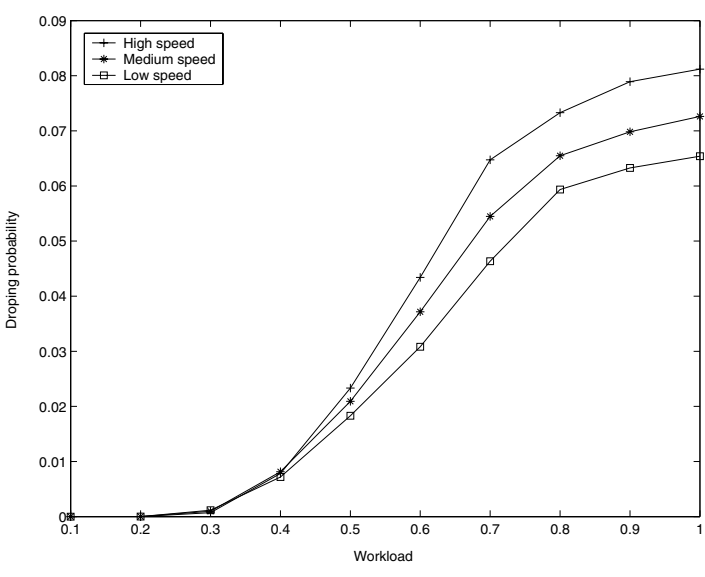

Fig. 9. Impacts on CDP by different moving speeds.

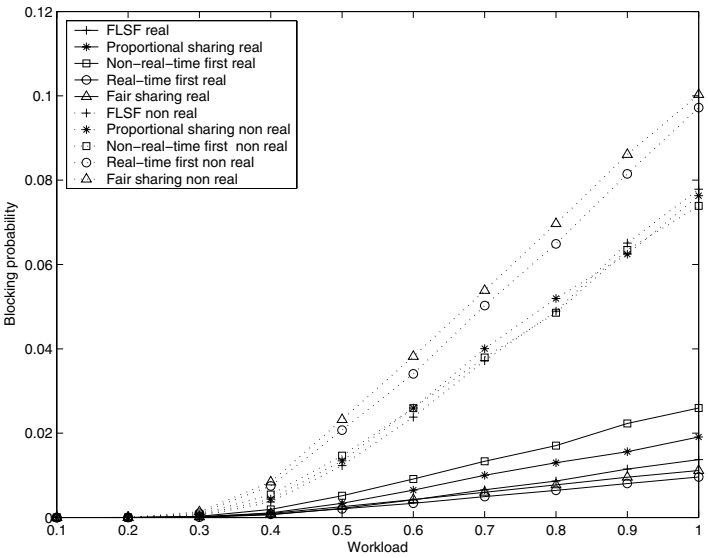

Fig. 10. CBPs for different resource reallocation schemes.

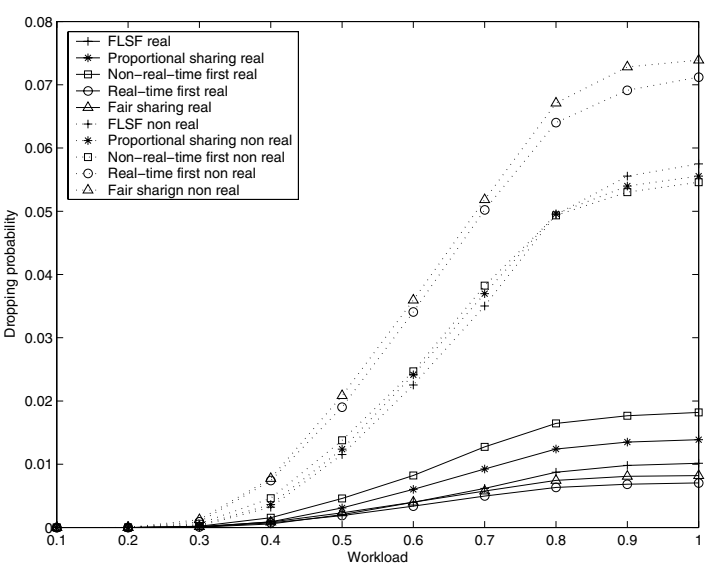

Fig. 11. CDPs for different resource reallocation schemes. 